\title{
Convective Weather Forecast Quality Metrics for Air Traffic Management Decision-Making
}

\author{
Gano.B. Chatterji" and Brett Gyarfas ${ }^{\dagger}$ \\ University of California Santa Cruz, Moffett Field, CA, 94035-1000 \\ and \\ William N. Chan ${ }^{\ddagger}$ and Larry A. Meyn \\ NASA Ames Research Center, Moffett Field, CA, 94035-1000
}

\section{Extended Abstract/Introduction}

\begin{abstract}
A ir Traffic Management decisions are often influenced by surface and enroute weather conditions. Surface visibility and wind conditions result in reduction of airport arrival rate and airport departure rate. Similarly, severe convective weather condition reduces the capacity of enroute airspace. Under such conditions, traffic flow management initiatives are employed to balance the demand against the remaining available capacity. Traffic flow management initiatives consist of ground stops, ground delay programs, miles-in-trail restrictions, rerouting and airborne holding. These initiatives eventually result in flight delays, which are detrimental to the airline operations. Of the various causes of delay, weather is often cited as the biggest cause; responsible for approximately $70 \%$ of the delays in the National Airspace System (NAS). ${ }^{1}$ Unusual operations due to weather sometime cause schedule disruption to the extent that it takes several days for the airline to recover from it. In addition to the operational consequences, severe weather also poses a risk to the aircraft itself. Since weather plays such a dominant role in aviation, airlines and air traffic service provider use meteorological forecasts, pilots are briefed about weather prior to and during flight, and aircraft are instrumented with weather radar.

With the availability of numerical weather prediction models, advent of faster computers and communications networks, a variety of convective weather forecasts are produced operationally and distributed by the National Center for Atmospheric Research (NCAR) and the National Weather Service (NWS). The operational National Convection Weather Detection (NCWD) product and the National Convective Weather Forecast (NCWF) product are provided by NCAR. The Collaborative Convective Forecast Product (CCFP) and Convective Significant Meteorological Advisories (C-SIGMET) forecasts are issued by the Aviation Weather Center (AWC) of the NWS.

The NCWD product provides observed ongoing convective activity data in a grid using a combination of the Next Generation Radar (NEXRAD) Vertical Integrated Liquid (VIL) product and lightning data. The NCWF product provides one-hour and two-hour forecasts of convective activity. These data are also reported in a grid and are updated once every five minutes based on radar observations and cloud-to-ground lightening data. The CCFP product is produced through a collaborative process between the AWC, airline and Air Route Traffic Control Center (ARTCC) meteorologists, and meteorologists from the Meteorological Service of Canada. CCFP consists of areas that are forecast to experience intense convection and thunderstorms. Areas of size 3000 square miles or larger containing at least $25 \%$ convection with at least $40 \mathrm{dBZ}$ composite reflectivity with echo tops of 25,000 feet and higher are denoted by polygons in CCFP. Once every two hours, two-hour, four-hour and six-hour forecasts are generated. C-SIGMET, which is generated by forecasters at AWC, is issued hourly in a text format and is valid for up to two hours. Regions with ongoing severe convective weather activity or regions where it is forecast to occur within 30 minutes are shown enclosed in polygons. The forecast regions are 3000 square miles or larger with at least $40 \%$ severe weather footprint.
\end{abstract}

\footnotetext{
*Principal Scientist and Task Manager, U. C. Santa Cruz, MS 210-8, Associate Fellow.

${ }^{\dagger}$ Graduate Student, U. C. Santa Cruz..

$\ddagger$ Aerospace Engineer, NASA Ames Research Center, MS 210-8.

$\S$ Acting Chief, AFM Branch, NASA Ames Research Center, MS 210-10, Associate Fellow.
} 
As interest in the forecast products has increased, forecast accuracy has become a topic of several investigations. Forecast accuracy is important both from the perspectives of the forecasters and of those who use the forecasts for their decision-making. Reference 2 describes a statistical verification method based on measures of forecast quality derived from a $2 \times 2$ classification table, with elements consisting of numbers of grid locations correctly predicted, and grid locations incorrectly predicted (missed alerts or false alarms). Reference 3 describes an alternative method for computing quality metrics based on the differences seen in the structures of the forecast regions and observed regions. The technique consists of identifying and grouping adjacent regions that appear to be part of the same weather system in the forecast data and finding a corresponding grouped region in the observation data. The difference in the properties of the two identified regions, for example the position of the centroids, then provides measures of forecast accuracy. This technique is very similar to "feature correspondence" techniques used in computer vision.

Since numerical weather prediction models are unable to accurately forecast the severity and the location of the storm cells several hours into the future when compared with observation data, there has been a growing interest in probabilistic description of convective weather. The classical approach for generating uncertainty bounds consists of integrating the state equations and covariance propagation equations forward in time. This step is readily recognized as the "process update" step of the Kalman Filter algorithm. ${ }^{4}$ The second well known method, known as the Monte Carlo method, consists of generating output samples by driving the forecast algorithm with input samples selected from distributions. The statistical properties of the distributions of the output samples are then used for defining the uncertainty bounds of the output variables. This method is computationally expensive for a complex model compared to the covariance propagation method. The main advantage of the Monte Carlo method is that a complex non-linear model can be easily handled. Recently, a few different methods for probabilistic forecasting have appeared in the literature. A method for computing probability of convection in a region using forecast data is described in Ref. 5. Probability at a grid location is computed as the fraction of grid points, within a box of specified dimensions around the grid location, with forecast convection precipitation exceeding a specified threshold. The main limitation of this method is that the results are dependent on the chosen dimensions of the box. The examples presented Ref. 5 show that this process is equivalent to low-pass filtering of the forecast data with a finite support spatial filter. References 6 and 7 describe the technique for computing percentage coverage within a $92 \times 92$ square-kilometer box and assigning the value to the center $4 \times 4$ square-kilometer box. This technique is same as that described in Ref. 5. Characterizing the forecast, following the process described in Refs. 5 through 7, in terms of percentage coverage or confidence level is notionally sound compared to characterizing in terms of probabilities because the probability of the forecast being correct can only be determined using actual observations. ${ }^{8}$ References 5 through 7 only use the forecast data and not the observations. The method for computing the probability of detection, false alarm ratio and several forecast quality metrics (Skill Scores) using both the forecast and observation data are given in Ref. 2.

This paper extends the statistical verification method in Ref. 2 to determine co-occurrence probabilities. The method consists of computing the probability that a severe weather cell (grid location) is detected in the observation data in the neighborhood of the severe weather cell in the forecast data. Probabilities of occurrence at the grid location and in its neighborhood with higher severity, and with lower severity in the observation data compared to that in the forecast data are examined. The method proposed in Refs. 5 through 7 is used for computing the probability that a certain number of cells in the neighborhood of severe weather cells in the forecast data are seen as severe weather cells in the observation data. Finally, the probability of existence of gaps in the observation data in the neighborhood of severe weather cells in forecast data is computed. Gaps are defined as openings between severe weather cells through which an aircraft can safely fly to its intended destination.

The rest of the paper is organized as follows. Section II summarizes the statistical verification method described in Ref. 2. The extension of this method for computing the co-occurrence probabilities in discussed in Section III. Numerical examples using NCWF forecast data and NCWD observation data are presented in Section III to elucidate the characteristics of the co-occurrence probabilities. This section also discusses the procedure for computing the probabilities that the severity of convection in the observation data will be higher or lower in the neighborhood of grid locations compared to that indicated at the grid locations in the forecast data. The probability of coverage of neighborhood grid cells is also described via examples in this section. Section IV discusses the gap detection algorithm and presents a numerical example to illustrate the method. The locations of the detected gaps in the observation data are used along with the locations of convective weather cells in the forecast data to determine the probability of existence of gaps in the neighborhood of these cells. Finally, the paper is concluded in Section V. 


\section{References}

${ }^{1}$ Krozel, J., Capozzi, B., Andre, A. D., and Smith, P., "The Future National Airspace System: Design Requirements Imposed By Weather Constraints," AIAA-2003-5769, Proceedings of AIAA Guidance, Navigation, and Control Conference, Austin, TX, August 11-14, 2003.

${ }^{2}$ Mahoney, J. L., Kucera, P., Hart, J. E., and Brown, B. G., "Quality Assessment Report National Convective Weather Forecast (NCWF-2)," submitted to Aviation Weather Technology Transfer Technical review panel, February 29, 2004, URL: http://www-ad.fsl.noaa.gov/fvb/publications/articles/NCWF-2-AWTT-Final.pdf.

${ }^{3}$ Brown, B. G., et. al., "New Verification Approaches for Convective Weather Forecasts," Proceedings of American Meteorological Society $11^{\text {th }}$ Conference on Aviation, Range and Aerospace Meteorology, Hyannis, MA, October 4-8, 2004,

${ }^{4}$ Gelb, A. (ed.), Applied Optimal Estimation, The M. I. T. Press, Massachusetts Institute of Technology, Cambridge, MA 02142 .

${ }^{5}$ Weygandt, S. S., and Benjamin, S. G., "RUC Model-Based Convective Probability Forecasts," Proceedings of American Meteorological Society $11^{\text {th }}$ Conference on Aviation, Range and Aerospace Meteorology, Hyannis, MA, October 4-8, 2004, URL: http://cdm.metronaviation.com/Workgroups/WxApps/related docs/ARAM5.3 SteveWeygandt.pdf.

${ }^{6}$ Mahoney, J. L., et. al., "Defining Observation Fields for Verification of Spatial Forecasts of Convection," Proceedings of 17th Conference on Probability and Statistics in the Atmospheric Sciences, Seattle, Washington, January 11-15, 2004, URL: http:/www-ad.fsl.noaa.gov/fvb/publications/articles/define ob fields 2004.pdf.

${ }^{7}$ Mahoney, J. L., et. al., "Defining Observation Fields for Verification of Spatial Forecasts of Convection: Part 2," Proceedings of American Meteorological Society $11^{\text {th }}$ Conference on Aviation, Range and Aerospace Meteorology, Hyannis, MA, October 4-8, 2004, URL: http://www-ad.fsl.noaa.gov/fvb/publications/articles/ARAM04-mahoney-final.pdf.

${ }^{8}$ Weather Applications Workgroup, "Statement of User Needs Collaborative Convective Forecast Product (CCFP-2005)," Version 8.7.1, January 27, 2005, URL: http://cdm.metronaviation.com/Workgroups/WxApps/CCFPUserNeeds05(V8.71)01-2705.doc. 